\title{
Romano-baskische Namen des Wiesels
}

(zu Zeitschr. '10, 215 Anm.).

Andereder (-rr) „Wiesel" bei den franz. Basken, eig. "schöne

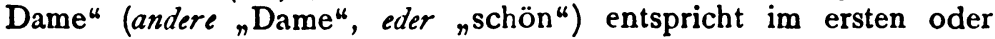
zweiten Teile einer Reihe von Wieselnamen der romanischen und anderer Sprachen; so würde eine Zusammensetzung der beiden in Italien üblichen Namen, des schriftgemälsen donnola und des mundartlichen *bellula ein genaues Synonym von andereder darstellen, oder vielmehr von dessen noch beliebterem Deminutiv, das durch Mouillierung der beiden $d$ gewonnen wird: andereder, -ar, anyereijer, anyereyer, anderiar (dieses lab. von Bidart, nach Darricarrère). Die Mouillierung kann aber auch blofs in einem Teile eintreten, im ersten: anyereder, angereder, oder im zweiten: and(e)rejer, andreyer, andereiger (gewifs handelt es sich zum Teil nur um verschiedene Schreibungen). Am weitesten hat sich von der Stammform entfernt: anjijar (dieses lab. von St.-Jean-de-Luz, nach Guilbeau Hitztegiko pasarte batzuek '08). Die ursprüngliche Bedeutung des Ausdrucks ist also allmählich ganz verdunkelt worden (wie z. B. auch beim ital. *bellula) und es frägt sich ob die mythischen oder abergläubischen Vorstellungen auf denen er beruht, bei den Basken noch fortdauern. Solche sind $z u$ ihnen entweder für sich verpflanzt worden oder zugleich nit der innern Sprachform, schwerlich diese allein, sodafs eine mehr oder weniger gedankenlose Nachbildung vorläge. Wenn mit andereder sich das im Gaskognischen vorkommende dauno-bero o. ä. völlig deckt, so hat natürlich Übertragung stattgefunden und es werden die Romanen die Empfänger gewesen sein. Denn das letztere Wort ist wenig verbreitet; Rolland führt es nur aus Bayonne an und im Atlas ist es an zwei andern, aber auch von der baskischen Sprachgrenze nicht allzuweit entfernten Punkten eingetragen.

Satandera bizk. guip., satandre niedernav. ronc., nach $\operatorname{Pr}$. Bonapartes Hdss. auch salaz., demin. satandre ronc. "Wiesel", eig. "Mäusedame" (Azkue: „señora de ratones; dame des souris"); satsteht für *sagut- (= sagu "Maus"; wegen des -t- vgl. meine Iber. Dekl. S. 62 f. Zeitschr. 'o9, 466), wie in sat-abia "Mäusenest", satarte "Mausefalle". Welchen Sinn kann nun diese Zusammensetzung haben? Das Wiesel ist einerseits ein geschworener Feind aller Mäuse und Ratten; anderseits gehörte es für Altertum und 
Mittelalter selbst in das weite Reich der Mäuse hinein.1 Manche Benennung des Wiesels, wie mlat. mus longus, longa, südfranz. (alp.) musset, sav. mozëla, lothr. musatte (vgl. franz. muset, -ette "Spitzmaus“) mögen aus einem Milsverständnis von mustela erwachsen sein, das doch "Mäusefängerin" bedeutet haben wird. Aber es heilst auch ${ }_{\text {"Ratte }}$ : auv. ra-moustiaou und danach moustiaou (männl.); ist das mallork. mostel (männl.) ähnlich zu erklären? Ebenso wird bei den Arabern, ohne dafs eine Lautähnlichkeit mitspielte, das Wiesel als Ratte (oder Maus) aufgefafst und zwar als „Pferderatte": maghreb. fär (auch weibl. $f \bar{a} r t$ ) el haail. Vielleicht hat das seinen Grund in dem was in Brehms Tierleben ${ }^{3}$ I, 614 vom Wiesel berichtet wird: „Auch in den Beinen von vorübergehenden Pferden hat es sich festgebissen und konnte nur durch vereinte Anstrengung von Rols und Reiter abgeschüttelt werden." Was nun and(e)ra, -e anlangt, welche seiner Bedeutungen sollen wir für satand(e)ra, $-e$ annehmen? Die ursprüngliche ist offenbar: „junges Weib" im allg., in welcher es aus dem Gallischen (*andera) entlehnt wurde (vgl. ir. ainnir "junges mannbares Mädchen "); dann "junge verheiratete Frau", „Frau“ im allg., „Dame“. Übersetzt man es nun hier mit „Dame“, so darf man an madj. hölgy errinnern, welches "Dame“ und "Hermelin" (Tier) bedeutet; in letzterem Sinn gesellt es sich auch dem Worte für „Wiesel“ zu: hölgymenyet „Hermelin". Dieses menyét (es ist eine nicht ganz klare Ableitung von meny "Schwiegertochter", älter "Braut", ,junge Frau") bezeichnet ebenfalls schon ein weibliches Wesen; anderseits wird in der Volkssprache dem menyét, ohne dafs der Sinn verändert würde, asssony „Frau" angehängt: menyet-asszony "Wiesel“ (nach Analogie von meny-asszony "Braut" neben meny). Aber welche Vorstellung liefse sich mit "Mäusedame" verbinden? Eine solche würde erst eintreten wenn wir „Dame“ durch „Herrin"“ ersetzten, und es wäre dafür nicht blols auf den georgischen Wieselnamen „Königin“ zu verweisen, sondern auf weit näherliegendes, nämlich darauf dafs die Bienenkönigin in der Soule andere heifst ${ }^{2}$ und hier wie auch sonst im franz. Baskenland so gerufen wird, ja mit dem Namen des Wiesels selbst: anderejer. Nur müssen wir gleich eingestehen dafs sich zwischen der Stellung der Königin im Bienenstaat und dem Verbältnis des Wiesels $2 u$ den Mäusen keinerlei Ähnlichkeit zeigt. Eher vermögen wir uns etwas $z \mathfrak{u}$ denken wenn wir ancera in satandera mit „Braut" oder ,junge Frau“ übersetzen - den Unterschied $z$ wischen beidem werden wir wohl bei einem Tiere vernachlässigen dürfen; das Wiesel wäre eine Art weiblicher Blaubart, es brächte allen die sich um der Liebe willen mit ihm einliefsen, in

1 Auch seine Verwandten werden oft als Ratten bezeichnet, z. B. der Iltis, bei uns Ratz; umgekehrt ist dessen oder des ihm nahe stehenden Frettchens Name: port. touräo, gal. touron (\} * putorione nach Carolina M. de Vasconcellos Rev. Lus. 3, 187) auf die Feldmaus: span. turón übergegangen. Nach dem Nuevo Dicc. von M. de Toro ('Or) ist der turón ein der Ratte ähnliches, stinkendes Tier, nach dem Wtb. der Akademie ${ }^{8}$ (79) der Dachs.

2 Im Labourd bedeutet andre auch den König unter den Kegeln. 
irgend einer Weise den Tod. Dals andera in satandera die eben angegebene Bedeutung hat, wird nun durch das spanische Arabisch bestätigt, in welchem seit dem 12. Jhrh. als Name des Wiesels 'arūsat al fïrān (oder vielmehr mit der Imale: fïrīn) d. i. „Braut der Mäuse" bezeugt ist. $\mathrm{Da}$ Arabisch und Baskisch in keine unmittelbare Berührung gekommen sind, so mufs das Spanische zwischen den beiden ganz gleichwertigen Ausdrücken vermittelt haben; vielleicht hält sich noch irgendwo ein novia de ratones o. ä. versteckt. Die Entstehung dieses Namens denke ich mir nun folgendermalsen. Einem mittelgr. $\nu v \mu \varphi i \tau \zeta \alpha$ oder wahrscheinlich schon altgr. $\nu \dot{v} \mu \varphi \eta$ („Braut"), „Wiesel" hat das arab. 'arüsa, 'arisa (auch ägypt.-arab. 'irsa, 'arsa) „Braut", „,junge Frau" die Bedeutung "Wiesel" entlehnt; und wiederun beruht die arab. Bezeichnung eines gewissen Meerfisches (chaetodon maculosus): 'arüsat el bahr "Meerwiesel" auf gr. $\gamma \alpha \lambda \tilde{\eta}$ oder lat. mustela. Die Araber brachten den fremdartigen Ausdruck in eine ihnen sehr geläufige Form: ibn 'irs (soviel wie „Sohn des Bräutigams"?), mit weibl. Plural: benāt 'irs; ob nicht auch bei ihnen irgend eine Sage oder ein Aberglauben mit dem Wiesel verknüpft ist, wäre freilich erst zu untersuchen. Jedenfalls ist es sehr begreiflich dafs man sich die Frage vorlegte: von wem ist das Wiesel eigentlich die Braut oder Frau? und dafs man das Bedürfnis fühlte den Ausdruck zu ergänzen. Dieses wurde in Spanien befriedigt; man fand dafs das Wiesel mit keinen andern Tieren mehr zu tun hätte als mit den Mäusen, und man verlobte oder verheiratete es scherzhafter Weise mit ihnen. Oder, was wohl noch eher anspricht, es vermischte sich der arabische Name mit einem romanischen "Mäusetöter" o. ä., wie er sich ja auch der äufsern Form nach mit einem romanischen Deminutiv (schon in mustella neben mustela vorgebildet) vermischte: "arūsalla (zu lesen: 'arūsilla) "Wiesel" steht in dem Leidener Glossar das im 12. Jhrh. nach einer nicht viel älteren Vorlage in Spanien geschrieben worden ist.1 In Sizilien konnten die romanischen Namen des Wiesels durchaus nicht auf den Gedanken bringen dals es verheiratet oder $z \mathrm{u}$ verheiraten sei und auf die Frage warum dies; dafür muls das arab. 'arūsa usw. verantwortlich gemacht werden. „Ha una particolar avidita per le galline e pel pollame in genere. Ora, perchè riesca innocua, bisogna maritarla, e si marita dicendole ad alta voce:

Si si' fimmina, ti dugnu lu figghiu di lu re;

Si si' màsculu, ti dugnu la figghia di la riggina.

Dopo questo matrimonio (che, in fondo, è uno scongiuro), la donnola non danneggerd più il pollame (Palermo, Marsala, Sala-

1 Aus welchem Grund Dozy Suppl. zu dem aus Bocthor entnommenen "arisa furet" mit einem Fragezeichen das Deminutiv hinzusetzt: "uraisa nWieselchen", weifs ich nicht; das Frett ist ja beträchtlich grölser als das Wiesel, auch ist überhaupt eine so ausgedrückte Unterscheidung beider Tiere unwabrscheinlich. 
paruta); ma invece sfogherd la sua avidità coi topi" (Pitrè Usi ... del pop. sic. 3, 440 f.). Schliefslich ist zu erwähnen dafs auch die Gelehrten des Altertums die Verbindung zwischen Wiesel und Maus für möglich hielten, erklärten sie doch das Wort $\mu v \gamma \alpha \lambda \tilde{\eta}$ "Spitz-

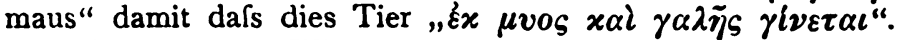

Ogigaztai, -ae „Wiesel“, eig. "Brot (und) Käse" ist nach van Eys und Azkue bizkaisch und scheint auf diese Mundart beschränkt zu sein (Käse heifst guip. gazta und franz.-bask. gazna, gasna). Das Wort steht schon im Modo breve de aprender la lengua Vizcayna, von R. Micoleta, Pritester zu Bilbao I653 (Ausgabe von Sevilla 1897 S. 13) als oguigastoea; oe (das z. B. in xamoea ebd. S. 18 ; span. jamón berechtigt ist) wird für ae verdruckt sein. Das Wiesel ist auf der Oberseite rötlich-braun, auf der Unterseite weifs gefärbt; diese Zweifarbigkeit fällt hinlänglich auf dafs das Tier danach benannt werde, und O. Keller (Die antike Tierwelt I, 165) nimmt in der Tat für $\gamma \alpha \lambda \tilde{\eta}$ „Wiesel" als Urbedeutung: "mehrfarbig" an. Auf diese Eigentümlichkeit habe auch ich Zeitschr. '99, 192 das bask. ogigastai bezogen. Ich kann hierfür keine Quelle mehr finden; war es meine eigene Vermutung, so sind andere auf die gleiche gekommen. F. Baráibar y Zumárraga Vocabulario de palabras usadas en Álava, Madrid 'o3 S. 196 sieht die Ursache dieser Benennung entweder in einer gewissen Neigung das Tier zu verherrlichen (?) oder nen cierta semejanza entre los colores pardo y blanco de su piel y los de la corteza y el meollo del pan y del queso". Er bemerkt dies bei Gelegenheit des dem ogigaztai, von der Endung abgesehen, genau entsprechenden alav. paniquesilla. Azkue Dicc. II, $97^{\mathrm{c}}$ weist pan y queso "Wiesel“ (ohne Erklärung) in dem Spanischen der Roncaler nach, die aber in ihrem Baskisch satandre sagen. Da also die spanische Bezeichnung am südwestlichen und südöstlichen Rande des baskischen Sprachgebietes auftritt, so liegt es nahe sie für eine Nachbildung der baskischen zu halten. Indessen gibt es verschiedene Umstände die zugunsten eines umgekehrten Verhältnisses sprechen. Zunächst begegnet uns paniquesa "Wiesel ${ }^{\text {" }}$ (man beachte hier, wie in paniquesilla, das weibliche Geschlecht -als ob man sich das Wiesel gar nicht männlich denken könnte) noch im Aragonischen, wo es, nach Borao, auch auf lebendige, bewegliche Mädchen und Buben übertragen wird. Offenbar ist es allgemein aragonisch, nicht etwa auf die ans Baskische storsende Nordwestecke beschränkt; aber aus dieser ist es allerdings in das Bearn und das Bigorre eingedrungen: über den altberühmten Aspepass nach Oloron, über den Pass von Anéou (hier wird der freundschaftliche Verkehr zwischen den Hirten der beiden Länder gerühmt) in das Ossautal und dessen Nachbarschaft (Eaux-Bonnes), über den Pass von Marcadaou nach Cauterets oder über die Rolandsbresche nach Gavarnie, Gèdre, Villelongue, bis nach Argeiès (auch nach Arrens in einem Nebental), schliefslich in die Ebene auf der rechten Seite des Gave de Pau, nach Arthez, Morlaas, Lembeye: païkezo, pankezo, pakezo (auch -e-; -a, -e), auch panghe 
neben panghizo im Argelèstal, alles nach Ausweis des Sprachatlas und von Rolland Faune pop. 7, 120 . Von Westen, aus dem Baskischen, kann das Wort diesen gaskognischen Mundarten nicht zugekommen sein, da die französischen Basken das "Brot und Käse" nicht kennen und die Gaskogner "Käse" nicht mit kezo ausdrücken. Aus Spanien scheint auch das coumayrelo "Wiesel" (span. comadreja) zu stammen welches aus den Départements Haute-Garonne, Ariège und Tarn verzeichnet wird. Da wie gesagt die Gaskogner den zweiten Teil von paniquesa als fremdartig empfanden, so ersetzten sie ihn durch einen andern und verbesserten damit zugleich den ganzen Ausdruck; "Brot und Milch" eignet sich noch mehr als "Brot und Käse" "für die Farben des Wiesels. Vielleicht aber wurde schon auf spanischer Seite pan $y$ leche gesagt; wenigstens erklärt sich lautlich daraus pallets(e), zum gask. leit stimmt panlett, pallett, an pakezo angeglichen ist pallezo - alle diese Formen fallen in das Dép. Hautes-Pyrénées. Im Osten schliefst sich nun eine dritte, wohl die jüngste Formenreihe an. Das pankezo war hier gar nicht mehr verstanden worden und hatte einen ganz äufserlichen Wandel durchgemacht, dessen Ursachen für mich dunkel sind: pankero (-éro), hauptsächlich im Dép. Haute-Garonne (bis Carbonne) und in der Südwestecke des Dép. Ariège (auch zu Nestier, H.-Pyr.). Der Ubergang des $z$ (das auf eine nicht sehr späte Entlehnung hinweist) in $r$ stimmt nicht zu den Lautgewohnheiten jener Gegenden; anderseits nehme ich kein Wort wahr das sich eingemischt haben könnte. Pankerre hat Rolland, ohne nähere Angabe, als bearnisch, nach Lespy, der aber im Wtb. panquêre schreibt; aus Morlaas, aber neben pakize, wird es von einem Helfer Rollands bezeugt. Weit nördlich von allen diesen Formen, zu Lectoure (Gers) findet sich pancarro; hier ist der Einfluls unverkennbar von lang. gask. missarro, micharro "Bilch" (diese Formen kommen in den benachbarten Départements Tarn-et-Garonne und Haute-Garonne vor). Mit pantero (zu Saint-Girons, Ariège) ist der dunkle Name wieder in ein helles Licht gerückt worden. ${ }^{1}{ }_{n}$ Brot $^{4}$

1 Bei der Erwägung der oben besprochenen gaskognischen Formen mit pa. war ich zuerst durch andere romanische gleichen Anlautes auf falsche Fährte gebracht worden. Diese selbst harren zum Teil noch der Deutung. Flechia Arch. glott. ital. 2, 50 setzt richtig siz. baddottula gleich *bellottula; aber er erklärt das $a$ nicht, und so besteht auch Pasqualinos Gleichsetzung des Wortes mit ballottola, pallottola zu Recht, kurz, dieses Wort hat sich mit dem andern vermischt. Deutlich gemacht wird dies besonders durch catan. badda allassata, obwohl ich glaube dals in der Wendung curriri comu badda allaszata es schon das Tier bedeutet (wir sagen: "wie ein Wiesel laufen"); die Glosse Trainas: "badda allazzata, forzata ${ }^{\star}$ ist mir nicht recht klar. Aus baddottula wurde zu" Messina paddottula; aus beddula an vielen Orten durch Einmengung von peddi ${ }_{n} \mathrm{Pelz}^{u}$ : piddotta, pidduottula (Pitre Usi sic. 3,440). Salvioni Rc. del R. Ist. Lomb. XL ('07), I 107 stellt sich die Entwicklung der siz. Formen etwas anders vor; aber wenn er sagt, das $e$ oder $i$ der ersten Silbe sei ${ }_{n}$ per ragioni tuttora ignote ${ }^{\mu}$ in $a$ übergegangen, so kann die Ursache doch nur in einem andern worte gesucht werden und das finden wir eben in balla, palla, dessen schliefsliche Beteiligung ja auch er zugibt. 
und "Käse" pflegen nun in allen Sprachen des Südens sich mehr oder weniger fest zu verbinden, besonders auch in sprichwörtlichen Redensarten, und wenn, nach Baráibar, die Bauern von A'lava sagen: „pan y queso saben á beso", so kann man hierin weder eine spanische, noch eine baskische Eigentümlichkeit sehen wollen. Das Gleiche gilt von einer solchen Verschmelzung wie sie im Verb paniquesear "an der Verteilung von Brot und Käse teilnehmen“ derselben Provinz (insb. der Hauptstadt Vitoria) angehört. Auch der übertragene Gebrauch von „Brot und Käse“ kann sich überall einstellen; so bezeichnet z. B. val. pa y formage ein mir nicht weiter bekanntes Kinderspiel. Dafs aber jene zoologische Bedeutung eher vom Spanischen ausgegangen ist als vom Baskischen, wird durch zwei botanische nahe gelegt, die dem Spanischen eignen, nicht so viel ich weifs dem Baskischen. Die Flügelfrucht der Ulme für welche die Römer den Kelten den Namen samara (-era) entlehnten, heifst nach Baráibar in A'lava paniquesillo, aber „en Segovia y en otras provincias castellanas" - also auch sehr fern vom baskischen Sprachgebiet - pan y queso. Sie wird von den Kindern gegessen.1 Aus paniqueso ist geworden arag. panichaza, so, nach Borao, in Borja und an andern Orten, während der verbreitetere Ausdruck dafür sopeta ist („episperma alar de la semilla de ciertos árboles $\delta$ sea hojuela que cae con la flor en la primavera"). Ein allgemein spanisches Wort aber ist pan y quesillo oder paniquesillo für das Hirtentäschelkraut, thlaspi bursa pastoris L., dessen dreieckig herzförmige Schotenfrucht sich einer Hirtentasche vergleichen lärst (span. [17. Jhrh.] camarilla Rolland Fl. pop. '2, 108, bedeutet eigentlich "kleine Hirtentasche aus Schaffell", hat also nichts mit lat. samara zu tun), pan y queso, pangüeso, panhueso für das grölsere, thlaspi campestre L. Die Hirtentasche enthält ja Brot und Käse; die Ulmenfrucht welche der des Hirtentäschelkrautes ähnelt (noch mehr aber der des grolsen Täschelkrautes, thlaspi arvense L.), hat wohl erst von diesem den Namen empfangen - man bemerke germanische Namen dieser Pflanzen wie Pfenniggras, penny-cress usw., ohne sich zu beeilen daraus Schlüsse zu ziehen.

Erbinucue, erbi-unide, eig. "Hasenamme" (erbi „Hase“, inude, unide "Amme") ist einer der span.-bask. Namen des Wiesels. R. Riegler (Wörter und Sachen 2, 186 ff.) hat in scharfsinniger Weise sich bemüht ihn zu erklären. Er verzichtet auf die Annahme einer an sich wohl denkbaren Verwechslung; da das Wiesel dem Hasen an die Kehle springt um sein Blut zu saugen, so mülste dieser „Wieselanme" heifsen. Durch die schottisch-englische Bezeichnung des kleinen Lappentauchers als "Hasenmutter“ angeregt, betrachtet er diese und die andere als gleichwertig mit "Hexe“ und knüpft an unsere Frau Holle an, die sich von Hasen die Lichter vorantragen

1 Im Süden sind diese Früchte der Ulme (ulmus campestris L.) vielleicht essenswert; bei uns werden sie nicht reif, wie ich aus $K$. Koch Die Bäume und Sträucher des alten Griechenlands S. 83 ersehe. 
lärst. Er gibt selbst zu dafs der Weg von der germanischen Gottheit zum baskischen Wiesel weit ist; ich fürchte, er ist zu weit. Im Spanischen heifst das Wiesel comadreja, was man mit nkleine Gevatterin " übersetzt; richtiger wäre "kleine Hebamme", denn *commater hat im Spanischen, wie im Südfranzösischen und Italienischen auch die Bed. "Hebamme". Jene Benennung könnte irgendwie mit der alten Sage zusammenhängen nach welcher die Galinthias oder Galanthis von ihrer Herrin, der Geburtsgöttin Eileithyia oder Lucina, zur Strafe für einen gewissen Frevel in ein Wiesel verwandelt wurde. Diesem romanischen Worte, mag es "Gevatterin" oder "Hebamme" bedeuten, entspricht gewifs das baskische „.... amme". Wie man nun bei der Bezeichnung des Wiesels als „Braut", „Frau“ fragte: von wem? und darauf die Antwort erfolgte: von Mäusen (Ratten), so auf die Frage: „Amme von wem?" die Antwort: „von Hasen", also beide Male wurden Tiere genannt die das Wiesel mit besonderer Lust verfolgt. Diese Zusammensetzungen würden demnach in ironischem Sinne zu nehmen sein. Eine andere Parallele zu erbiñude scheint mir durch bask. unť̌arta „Frettchen“ geboten zu werden; dieses Tier ist in Spanien weit volkstümlicher als das ihm verwandte Wiesel, da es dort schon seit alter Zeit, später auch anderswo zur Jagd des in besonderem Sinne spanischen Kaninchens (bask. un/si) benutzt worden ist. Für arta ${ }^{1}$ freilich weifs ich keine einleuchtende Erklärung; an eine Beziehung zu artu "nehmen" ist nicht zu denken ("Kaninchenfänger" wäre *untsartzaile). Sollte das Wort arta "Sorge" darin stecken, und dieses eine solche Begriffsentwicklung erfahren haben wie span. cura (zu *urator), sodals untšarta soviel wäre wie "Kaninchenpfleger"? Dies wird weniger unwahrscheinlich wenn, wie ich glaube, das Wort die volksetymologische Umdeutung eines andern ist; ja dann braucht überhaupt mit dem zweiten Teil gar keine eigene Vorstellung verknüpft zu sein, es hätte sich eben nur untsi eingemischt. Und zwar genauer gesagt: es wären diesem zulieb $r$ und $n$, umgestellt worden in einem *urtsanda, von dem mir weitere Nebenformen, in auf- oder absteigender Linie, zu sein scheinen urdanda „Wiesel", (kata)kuisantša (Larr.) 2, (kata) unsantš (Harriet hdsch.) „Hausmarder “, ursantš, uršints, (kata)purtšints, (kata)kušantša" usw. "Eichhörnchen", worüber ich Bask. u. Rom. S. 32 . 60 gesprochen habe; wenn Salaberry ursins mit "Wiesel" übersetzt, so ist das vielleicht kein Versehen wie ich

1 Lautete es arda, so dürfte span. port. arda, guip. (kat)arde „Eichhörnchen" erwogen werden.

2 Dieses -kuis- und -kus., wozu noch die von $\operatorname{mir}$ in B. u. $R$. schon angeführten katakusa und kutusa "Marder" kommen, müssen bei den von Gauchat S. 24 f. besprochenen lomb. Wortformen cusa, gusa, gosa usw. „Eichhörnchen", "Silch" noch berücksichtigt werden (wenn übrigens in gosmatela sich wirklich mustela eingemischt hat, so darf doch nicht übersehen werden dafs matela in gewissen berg. Mdd. soviel wie "Mädchen" ist). Und wie steht es mit bearn. causete ${ }_{n}$ Wiesel ", eig. ${ }_{n}$ Dinglein "? Vgl. einerseits bearn. causou, causilhou nkleines junges Mädchen", anderseits nürnb. schönes Dinglein "Wiesel". 
meinte. Über die Verwechslung der Namen beider Tiere siehe noch Gauchat Les noms gallo-romans de l'écureuil, S.-A. aus den Wilmotte-Mélanges S. 23 f. und S. 18 (lyon. beléla „Eichhörnchen "). 1 Der Parallelismus zwischen untšarta nnd erbiñude würde noch grölser sein wenn auch das letztere sich als eine Umbildung erwiese. Dafs es eine solche sei, ist zum mindesten nicht unwahrscheinlich. Ich kenne drei Nebenformen von erbiñude. Zuerst erbiñure, von Azkue aus Andoain (Guip.) angeführt, erbinuria (mit Artikel) ${ }^{2}$, mir auch aus dem ziemlich weit davon entfernten Zumaya (Guip.) bezeugt; bei dem im Bask. so gewöhnlichen Wechsel zwischen $r$ und $d$ (z. B. udo „Frettchen"' span. hurón) ist sie von geringem Belang. $\mathrm{Ob}$ auch in erbindori (Azkue nach Duvoisin; also vielleicht aus dem Labourd) eine nur lautliche Variante vorliegt, ist weniger sicher. Wechsel zwischen $n d$ und $\tilde{n}, n y$ ist mehrfach belegt, dann aber wird jenes meist das ältere sein. Endlich gibt es auch für arminyeri (so in Aezkoa; mir von G. Lacombe aus den Hdss. des Prinzen Bonaparte mitgeteilt) keine ernsten Bedenken gegen eine rein lautliche Entwicklung; $m$ entsteht, bei folgendem $n$, oft aus

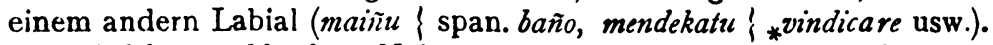
Aber infolge rückläufiger Neigung kann auch armin- zu erbin-geworden sein (wie lat. lamia \{ bask. lamiña, -ina \{ labina). Und wenn erbi- einen Sinn gibt, so auch armin-; man darf darin das span. armiño, prov. ermi(n) "Hermelin" erblicken, das im Bask. allerdings nicht sehr gebräuchlich geworden zu sein scheint (armiño, armin hat Larramendi und der ihm allzugetreue Fabre, armino Guilbeau Darricarrère und Azkue haben das Wort nicht aufgenommen, ja nicht einmal Aizkibel). Das Hermelin ist dem Wiesel sehr nahe verwandt und heifst deshalb auch das grofse Wiesel, das weifse Wiesel; so könnte ja umgekehrt das Wiesel als das kleine Hermelin, das rote Hermelin bezeichnet worden sein. ${ }^{3}$ Der Gedanke an ein bask. armin gorri "rotes Hermelin" scheint mir hier ausgeschlossen zu sein; aber ein dem nordfranz. herminette entsprechendes südfranz. *herminella, bearn. *erminhere wäre recht wohl möglich, aus dem sich bask. arminyeri usw. zwanglos ergäbe. Ermi- wurde als erbi-

'Ich möchte fragen ob sich mayenn. moussy $\hat{o}$ "Eichhörnchen" nicht an mustela (mlat. auch muscella, mussela) anknüpten "läfst; vgl. das in den pikardischen Mdd. herrschende moussouale "Wiesel". Sonst würde ich an eine mdi. Variante von monsieur denken, die auf das "Männchen" machende Eichhörnchen doch besser palst als eine Ableitung von einem Verb nerbergen". Auch dafs der Name timor rür "Eichhörnchen" in einer bergam. Md. sich, wie Gauchat meint, auf seine Furchtsamkeit bezieht, will mir aus äufsern und innern Gründen nicht recht eingehen.

2 In den meisten bask. Mdd. gebt das auslautende $e$ vor dem Artikel - $a$ in $i$ über, sodals da man die Substantive mit dem Artikel anzugeben pflegt, wir vielfach darüber in Zweifel geraten ob der Auslaut $e$ oder $i$ ist. Erbiñudi bei Riegler beruht abcr auf einem Versehen; auch mit dem Artikel wird erbiñudea geschrieben.

- So nennt man in Deutschen das Wiescl auch Hermännchen, Hermchen. In den Larraineko Albadak (volkstümlichen Ständchen zu Lichtmels), von Dodgson 1906 als Flugblatt herausgegeben heilst es: 
„Hase" mifsverstanden und -inhere entwickelte sich zu einem verständlichen iñude "Amme“. Zum Schlufs mache ich darauf aufmerksam dars wenn sich erbiñude auf das Wiesel wohl in schlimmem Sinne, so satandere in gutem Sinne bezieht. Denn das Tier wird sehr verschieden gewertet; je nachdem es dem Menschen Schaden oder Nutzen bringt, ist es gefürchtet und beliebt; man glaubt an manchen Orten dafs es Glück in die Häuser bringe und an andern betrachtet man es als Vorzeichen von Tod und Unglück. So befinden wir uns denn auch im Zweifel ob span. gal. garduña, port. gardunho „Hausmarder" (mustela foina L.) im ironischen oder im ernsten Sinn gemeint ist; denn ursprünglich kann es nichts anderes bedeutet haben als „Haushälter(in)“ (s. Zeitschr. '99, 19 I f.) oder besser „Haushüter(in)“. In einer Fabel des Phaedrus (I, 22) und seiner mittelalterlichen Nachfolger (s. Hervieux Les fabulistes latins II) fleht das Wiesel einen Mann der es gefangen hat, um Schonung an, indem es darauf hinweist dals es sein Haus von den lästigen Mäusen säubere; der Mann aber rechnet ihm das Schlimme das er von ihm erfahren hat, höher an und tötet es. In der portugiesischen Fassung des 15. Jhrhs. welche J. Leite de Vasconcellos aufgefunden und herausgegeben hat (O livro de Esopo, Lisboa 1906), gebraucht das Wiesel nicht einen Ausdruck der dem der lateinischen Texte (purgare, expurgare) entspräche, sondern „prometia-lhe de guardar bem toda ssua casa, que os rratos nom lhe fezessem dapno" (S. 26). Ich glaube hierin eine unwillkürliche Beziehung auf gardunho zu sehen, obwohl das Wiesel in dieser Fabel donesinha (gal. donicela, port. doninha) genannt wird. Es scheint mir um so mehr dafs auch jener Name ursprünglich von dem Wiesel gegolten hat, als dieses im Galizischen auch garridiña (von garrido "schön", „anmutig“) heifst, das ja zum franz. belette, ital. *bellula stimmt. Vielleicht ist garduña aus garridina (das dem Spanischen nur in der besondern Bedeutung fehlt) umgebildet worden; das mürste freilich in früher Zeit geschehen sein. Die Übertragung des Namens vom Wiesel auf den Hausmarder kann nicht befremden (altgal. donesynna wird vom Frettchen gesagt; s. Rev. Lus. 3, 187 Anm. 2); gal. garduña wendet man auch auf die Genettkatze an. Ob port. gardunha irgendwo in der Bed. "Dachs" vorkommt, welche für gardunia in jener Urkunde von 1126 angenommen wird (s. Zeitschr. '99, 192), habe ich immer noch nicht erfahren können. Obwohl dieses allerdings derselben Familie der Musteliden angehörige Tier schon durch seine Grölse aufserordentlich von den Wieseln und Mardern verschieden ist, finden doch auch sonst Namenverwechslungen mit ihnen statt;

Hourra houn da jateko Erbindolia hutchako.
La noisette est bonne à manger, L'hermine est bonne pour le bahut.

Die Úbersetzung ist von einem Souler (Larrau liegt auch in der Soule); es scheint in der Tat nichts anders möglich $z$ u sein als dafs erbindolia hier soviel bedeutet wie der Hermelinpelz". Wechsel von $l$ und $r$ ist im Bask. sehr häufig. [Korrekturnote.] 
von dem schon früher erwähnten südfranz. rabas abgesehen, vergleiche oben S. 161 Anm. I, und hier unten, sowie rum. dihor "marthe, fouine, blaireau" (bei R. de Pontbriant).

Mimitš "Wiesel“ von Azkue nach S. Pouvreau gebucht (auch bei Harriet hdsch.). Wir haben hier kosende Reduplikation wie in franz. mimi „Kätzchen“, und vielleicht bedeutet eigentlich das ganze Wort nichts anderes; mit milts-mits, mitsina wird im Baskischen die Katze gerufen. Doch erinnert es auch an bizk. misar (in den andern Mdd. musar) „Murmeltier", bizk. (kata)mišar, (kata)mišin „Eichhörnchen“, guip. lumišar, lab. lumisar, (wohl mit angewachsenem rom. Artikel) „Bilch", von südfranz. micharro u. ä. "Murmeltier und "Bilch" $\{*$ mus aranea (s. mein Bask. u. Rom. S. 32). Die Spitzmaus bekommt zuweilen den Namen des Wiesels: lang. (Aude) moustelo, altfranz. rablette d. i. rat belette (Rolland Faune pop. 7, 47 f.), wie umgekehrt (so mlat. migale $=\mu v \gamma \alpha \lambda \tilde{\eta}$; und s. oben S. 163).

Mustela „Wiesel", also ganz das lateinische Wort, führt Azkuc aus dem Bizk. von Markina an, nur mit der mir unverständlichen Erklärung: „comadreja, á diferencia de ogi-gaztae, que tiene blanco el pecho". Ferner gleichbed. muslerle aus dem Bizk. von Izpaster und Lekeitio. Eine ganz entsprechende Form, nämlich mousterlo findet sich im Südfranz. und zwar, dem Atlas zufolge, zu Vaucluse; sie ist aus der Vermischung der benachbarten Formen moustelo und mouslero entstanden und würde einen Beleg für den Vorgang bilden den Salvioni in burda buda + bura angenommen hat (Zeitschr. '11, 97). Das bask. musterle aber mufs, dem Orte seines Vorkommens gemäls, anders erklärt werden; in ihm ist mustela mit bizk. mierle „Edelmarder" (bei Larramendi unter garduña) verschmolzen. Dieses läfst sich schwerlich als *mariulus auffassen (das sonst in diesem Sinne als baskisch angeführte marte [Larr.], marterina [Fabre, Guilbeau] ist wohl ein junges Lehnwort; es fehlt bei Azkue im Wtb., aber nicht in dem von ihm mitverfarsten Manual de conversación '98 S. 275, wo übrigens mierle = pitosa als ,garduña" erklärt wird); ich denke eher an ein $*$ melulus von meles. Denn dafs lat. meles wegen des rom. melogna nur den Dachs, nicht den Marder habe bezeichnen können, das gebe ich nicht zu; in verschiedenen Gegenden mochte die Bedeutung des Wortes eine verschiedene sein (s. oben S. I68f.), besonders nun bei Anwendung einer Deminutivform. Mlat. melos, melo, melota u. ä. wird auch vom Hermelin gebraucht (vgl. Gauchat Les noms de l'écureuil S. 17). Bei der Untersuchung von mierle beachte man das fast homonyme bizk. mierlu „Fenchel", Nebenform von bizk. mielu, mieloi, hochnav. guip. bizk. milu, lab. milura, bei Fabre auch mâlu, was wohl irgendwie mit dem gleichbed.

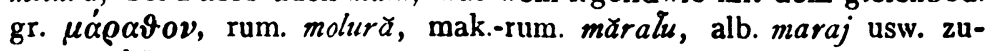
sammenhängt.

H. SCHUCHARDT. 